\title{
DEDUCCIÓN FÍSICA PARA UN MODELO DE UN SISTEMA PARCIALMENTE VISCOELÁSTICO
}

\author{
Alfonso Pérez Salvatierra \\ Universidad Nacional Mayor de San Marcos
}

\section{FUERZAS INTERNAS Y EXTERNAS}

Si ninguna fuerza externa es aplicada a un cuerpo, todas sus partes están en equilibrio mecánico. Si alguna perturbación debido a las fuerzas causa al cuerpo una deformación, entonces el equilibrio de las moléculas es afectado y el cuerpo no mas está en equilibrio mecánico; estos son producidos por fuerzas internas que tienden mantener el cuerpo en su estado original, como una forma de resistencia.

Las fuerzas internas tienen origen molecular directa y su rayo de acción es muy pequeño, se compara con las distancias consideradas en la teoría de la elasticidad; son generalmente consideradas como fuerzas que reactivan alguna perturbación realizada en la frontera del cuerpo.

Fuerzas que vienen del medio externo son llamadas fuerzas externas y son dividas en dos clases: fuerzas de cuerpo (o de masa) y fuerzas de contacto.

Si el cuerpo toma contacto con alguna fuerza del medio externo, entonces en la superficie de contacto aparecen fuerzas de corto alcance, también llamadas fuerzas de contacto (o fuerzas de superficie). Tales fuerzas decrecen extremadamente rápido como el crecimiento de la distancia entre los elementos de interacción.

Está claro que toda acción externa no puede ser representada por fuerzas de contacto. Gravedad, fuerza electromagnética, fuerza centrifuga son algunos de esos ejemplos. La acción de tales fuerzas en una partícula elemental del cuerpo es estáticamente equivalente a la fuerza aplicada en su centro de masa, es decir, las fuerzas y momentos producidos son proporcionales a la masa de la partícula bajo la cual ellas activan. Son llamadas fuerzas de cuerpo y momentos de cuerpo, respectivamente, y decrecen suavemente con el crecimiento de la distancia entre los elementos de interacción.

Resumiendo:

Fuerzas activando en un sólido = fuerza de cuerpo + fuerzas de contacto.

Donde las fuerzas de contacto pueden ser entendidas como fuerzas internas.

\section{LA TENSIÓN Y LA DEFORMACIÓN}

Introduciremos el concepto de stress para describir las fuerzas internas en un punto del cuerpo, que son definidas como fuerzas (o componentes de fuerzas) por unidad de área.

La deformación describe la medida de la intensidad de la deformación local en un punto. 
Considere $x=x_{j}$ la posición inicial de un punto $\mathrm{P}$ en el estado no deformado de un cuerpo y $\mathrm{X}=\mathrm{X}\left(x_{j}\right)$, la posición de $\mathrm{P}$ después de la deformación, transcurrido en un tiempo $\mathrm{t}(i, j=1,2,3)$. Tal transformación es continua y puede ser escrita como:

$$
X_{i}=X_{i}\left(x_{j}, t\right),
$$

esto es, la posición de $\mathrm{P}$ es función de la posición original y del tiempo transcurrido.

El vector desplazamiento $\quad u=u_{i}(i=1,2,3)$ relaciona las posiciones de $\mathrm{P}$ antes y después de la deformación:

$$
u=X-x \quad \text { o } \quad u_{i}=X_{i}-x_{i} \quad, \quad i=1,2,3 .
$$

Gráficamente tenemos:

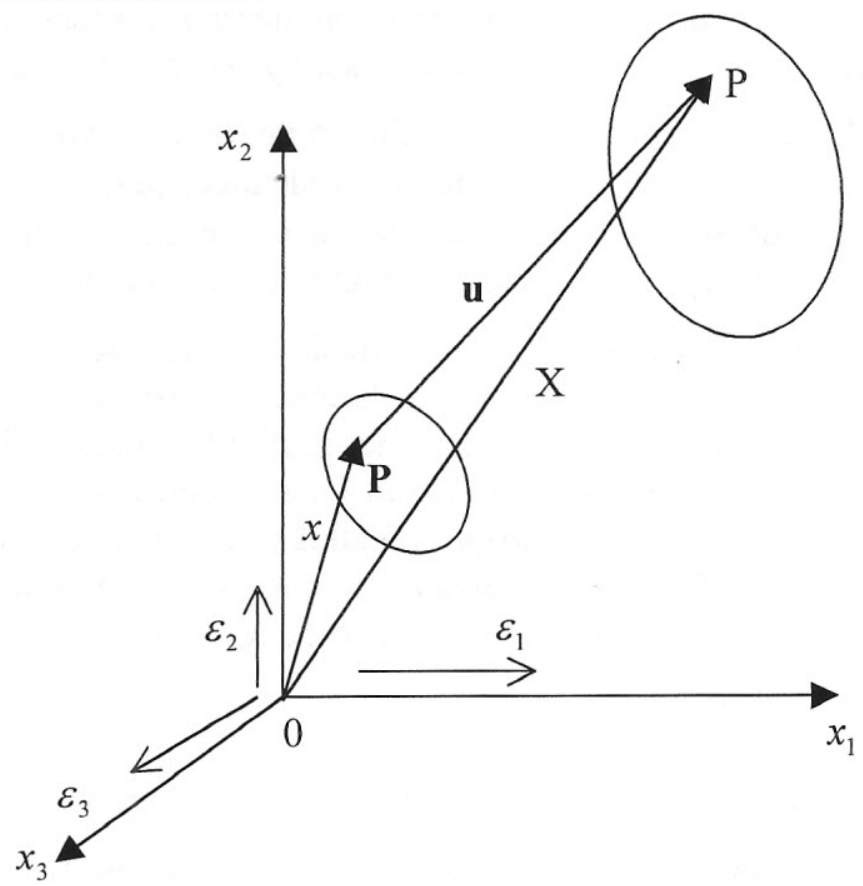

La deformación es dada por,

$$
\varepsilon_{i j}=\frac{1}{2}\left(\frac{\partial u_{i}}{\partial x_{j}}+\frac{\partial u_{j}}{\partial x_{i}}\right), \quad i, j=1,2,3 .
$$

La ecuación (1) es llamada ecuación deformación - desplazamiento. 
De forma análoga, para un análisis unidimensional, en un test de relajación de una deformación constante $\varepsilon(x, t)=\varepsilon_{0} G(t) \quad$ o $\quad G(t)=\frac{\sigma(x, t)}{\varepsilon_{0}}$.

La función $\mathrm{G}(\mathrm{t})$ así obtenida es llamada función de relajación (relaxation modulus), es la tensión por unidad de deformación aplicada. Las funciones $J(t), G(t)$ son propias de cada material. Si una deformación $\varepsilon_{o}$ es aplicada en $t=0$ en un material viscoelástico y entonces en $t=\tau_{1}, \varepsilon_{1}$ es aplicado, la tensión total será dada, en cualquier tiempo subsecuente a $\tau_{1}$ por la suma de las tensiones en cada tiempo correspondiente a cada tensión, como si actuasen separadamente, esto es,

$$
\sigma(x, t)=\varepsilon_{0} G(t) H(t)+\varepsilon_{1} G\left(t-\tau_{1}\right) H\left(t-\tau_{1}\right)
$$
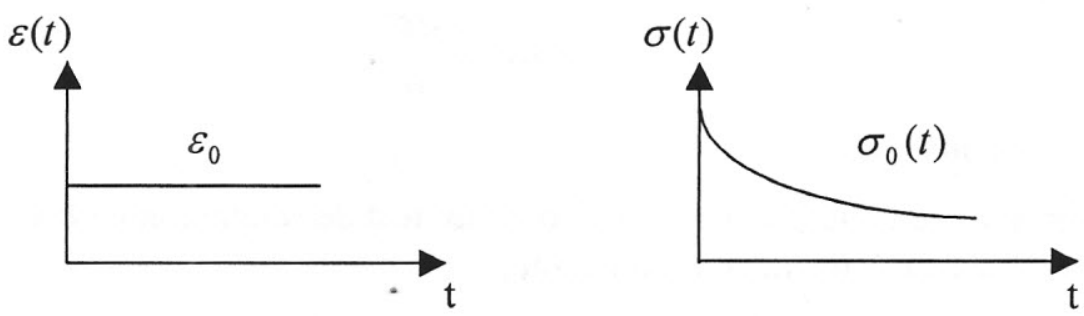

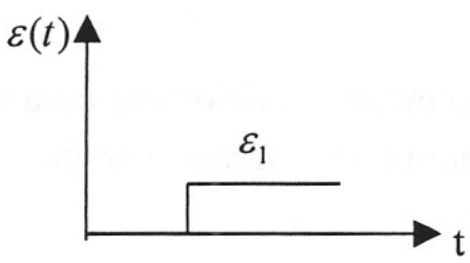

$\tau_{1}$

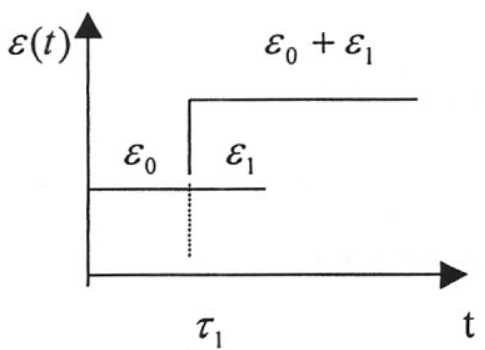

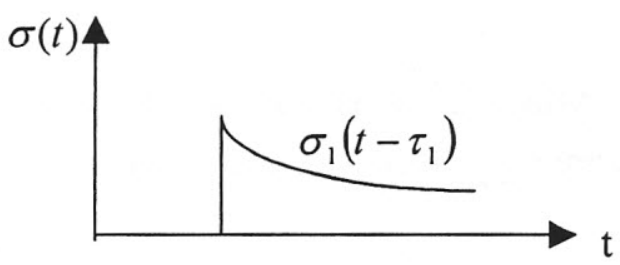

$\tau_{1}$

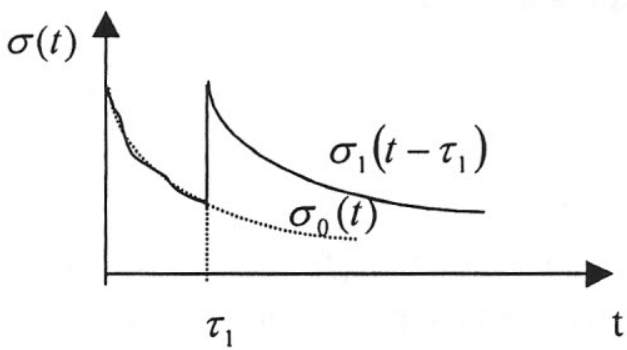

Esto es conocido como el Principio de la Superposición de Boltzmann [1], [3]

Materiales bajo tensión constante presentan una deformación suave y continua. A este proceso llamamos test de fluencia.

Usando la función de paso unitario, podemos escribir el test de fluencia histórico de $\sigma=0 \quad$ a $\quad \sigma=\sigma_{0}$ en $t=\tau_{0}$ como, 


$$
\sigma(x, t)=\sigma_{0} H\left(t-\tau_{0}\right)
$$

Definiendo ambos valores de tensión durante el test de fluencia y el tiempo de aplicación.

Durante un test de fluencia, siempre encontramos una componente elástica de deformación $\varepsilon^{e}$ (instantánea) y una componente creep strain $\varepsilon^{c}$ (atrasada). La deformación total $\varepsilon$, en cualquier instante $\tau$ en un test de fluencia es:

$$
\varepsilon=\varepsilon^{e}+\varepsilon^{c}
$$

y la tasa de deformación, $\dot{\varepsilon}$ es dada por

$$
\dot{\varepsilon}(x, t)=\frac{d \varepsilon^{c}}{d t}
$$

pues $\varepsilon^{e}$ es constante.

Consideremos, un cuerpo viscoelástico en un test de relajación (relaxation test), esto es, sometido a una deformación constante,

$$
\varepsilon(x, t)=\varepsilon_{0} H\left(t-\tau_{0}\right) .
$$

Midiendo la tensión $\sigma(x, t)$ necesaria para mantener la deformación a un valor constante $\varepsilon_{0}$, para $\tau_{0} \leq \tau \leq t$, tenemos que ella disminuye progresivamente.

En un test de fluencia, la tensión constante $\sigma(x, t)=\sigma_{0} H\left(t-\tau_{0}\right)$ es aplicada y la deformación $\varepsilon(x, t)$ es medida. Para materiales lineales, la deformación puede ser representada como,

$$
\varepsilon(x, t)=\sigma_{0} J(x, t) \quad \text { o } \quad J(x, t)=\frac{\varepsilon(x, t)}{\sigma_{0}} .
$$

La función $J(x, t)$ es llamada función de fluencia (creep compliance), esto es, el creep strain por unidad de tensión aplicada.

\section{LAS ECUACIONES CONSTITUTIVAS}

Las características físicas de cada material son especificadas por sus ecuaciones constitutivas. Una ecuación constitutiva es una relación entre fuerzas y deformaciones: fuerzas aplicadas a un cuerpo causan a él una deformación cuyo tipo de intensidad varia con la naturaleza del cuerpo. 
A un cuerpo aplicamos una tensión $\sigma(x, t)$ variando en el tiempo $\left(\tau_{0} \leq \tau \leq t\right)$ y medimos la deformación correspondiente $\varepsilon(x, t)$. Formalmente podemos escribir:

$$
\varepsilon(x, t)=D(\sigma(x, t)), \quad\left(\tau_{0} \leq \tau \leq t\right),
$$

donde $\quad D: C^{0}\left(\tau_{0}, t\right) \rightarrow R$.

Las experiencias también indican que en ciertos materiales la tensión en el punto $\mathrm{x}$ depende de toda la historia de la deformación. Así el aspecto característico de las propiedades viscoelásticas es esencialmente función del tiempo. En efecto, materiales bajo tensión constante aumenten su deformación con el tiempo, por lo menos próximo del punto de equilibrio, en cuanto que bajo deformación constante, la tensión decrece con el tiempo.

La función de paso unitario es definida como:

$$
\mathrm{H}\left(\mathrm{t}-\tau_{0}\right)=\left\{\begin{array}{lll}
1, & \text { si } t>\tau_{0} \\
1 / 2 & , & \text { si } t=\tau_{0} \\
0 & , & \text { si } t<\tau_{0}
\end{array}\right.
$$

donde $\tau_{0} \in R$ arbitrario y $\mathrm{t}$ el tiempo.

La historia de un proceso $f$ debido al tiempo $t$ es definido por:

$$
f(t)=f_{0} H\left(t-\tau_{o}\right)
$$

Vamos a considerar un cuerpo sólido A en equilibrio estático (aceleración nula o velocidad constante) y sea B una subversión del sólido sujeto a fuerzas de contacto sobre su superficie $\mathrm{S}$ y a las fuerzas de momento de toda la región $\mathrm{S}$. Considere $\mathrm{x}$ un punto interno del sólido, de coordenadas $x_{i}(i=1,2,3)$ perteneciente a la superficie de B. En x construimos un plano tangente $\pi$, con normal exterior $v$. Con relación a la región $\mathrm{A}, \pi$ tiene normal exterior $-v$ en $x$.

Observe que la región A ejerce una fuerza en la región $\mathrm{B}$ a través de $\pi$ en una vecindad de $x$.
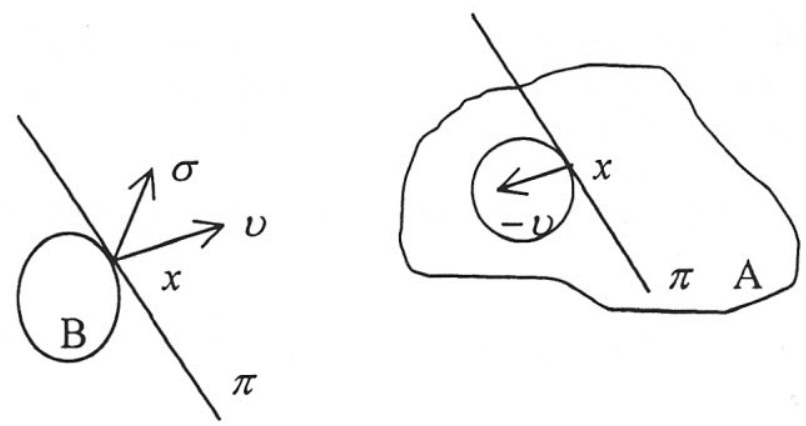
Sea $\Delta S$ una pequeña subversión de plano $\pi$ conteniendo el punto $x$. La resultante de las fuerzas alcanzado en $\Delta S$ causado por la acción de A sobre B es denotada por $\Delta F$ y el vector tensión $\sigma$ alcanzado en $x$ es definido por:

$$
\sigma=\lim _{\Delta S \rightarrow 0} \frac{\Delta F}{\Delta S}=\frac{d F}{d S}
$$

Nótese que, $\sigma$ tiene como variables a $x$ y $v$, esto es,

$$
\sigma=\sigma(x, v)
$$

y $\sigma$ describe todas las fuerzas o momentos transmitidos de un lado a otro de una sección de área $\Delta S$.

Si la tensión es arbitraria (variable como el tiempo), amenos de una constante, podemos aproximarla por la suma de una seria de deformaciones:

de donde

$$
\varepsilon(x, t)=\sum_{i=1}^{n} \Delta \varepsilon_{i}(x, t) H\left(t-\tau_{i}\right)
$$

$$
\sigma(x, t)=\sum_{i=1}^{n} \sigma\left(x, t-\tau_{i}\right)=\sum_{i=1}^{n} \Delta \varepsilon_{i}(x, t) G\left(t-\tau_{i}\right) H\left(t-\tau_{i}\right)
$$

Y si el número total de pasos tiende al infinito, la tensión total puede ser expresada como una integral:

$$
\sigma(x, t)=\int_{\tau_{0}}^{t} G(t-\tau) H(t-\tau) d(\varepsilon(x, \tau)) .
$$

Si la deformación histórica es diferenciable y como $\tau_{0} \leq \tau \leq t$, la función $H\left(t-\tau_{0}\right)$ en (2) es, por lo tanto, siempre la unidad. Luego podemos escribir como:

$$
\sigma(x, t)=\int_{\tau_{0}}^{t} G(t-\tau) \frac{\partial}{\partial \tau} \varepsilon(x, \tau) d \tau,
$$

donde $\tau$ es arbitrario y representa el tiempo pasado.

La integral (3) , llamada "integral hereditaria", fue primeramente presentada por Volterra. El núcleo de la integral, $\mathrm{G}(\mathrm{t}-\tau)$ es una función memoria, que describe la dependencia entre la deformación y la tensión.

Integrando por partes el segundo miembro de (3) obtenemos,

$$
\sigma(x, t)=G(0) \varepsilon(x, t)-\int_{\tau_{0}}^{t} \frac{\partial}{\partial \tau} G(t-\tau) \varepsilon(x, \tau) d \tau .
$$


Haciendo $-\frac{\partial}{\partial \tau} G(t-\tau)=g(t-\tau)$, tenemos:

$$
\sigma(x, t)=G(0) \varepsilon(x, t)-\int_{\tau_{0}}^{t} g(t-\tau) \varepsilon(x, \tau) d \tau
$$

La ecuación (4) es llamada ecuación constitutiva, “tensión-deformación”.

Observemos que: $\mathrm{G}(0)$ es una constante positiva, $-\frac{\partial}{\partial \tau} G(t-\tau)$ es positiva, integrable y por lo tanto, $G$ es decreciente y satisface:

$$
\mathrm{G}(0)-\int g(t-\tau) d \tau>0
$$

\section{BALANCE DEL MOMENTO LINEAL}

Las fuerzas actuando en un material o en sus parte son divididas en fuerzas de cuerpo $\mathrm{y}$ fuerzas de contacto. Consideremos un sólido que ocupa un volumen $\mathrm{V}$ en un tiempo t. Sea $F=F(x, t)$ la fuerza de cuerpo por unidad de masa y $\sigma=\sigma(v, t)$ la fuerza de contacto por unidad de área en su superficie $\mathrm{S}$, donde $v=v(x)$ es el vector normal a S. La ecuación para el balance de momento lineal es dado por:

$$
\frac{d}{d t}\left(\int_{V} \rho v d V\right)=\int_{S} \sigma(v, t) d S+\int_{V} \rho F d V=\int_{V} \rho d v d V
$$

donde $\rho$ es la densidad lineal de masa y vamos asumir que la masa es conservada tal que

$$
\frac{d v}{d t}=\frac{d^{2}}{d t^{2}} u
$$

es la aceleración. Notemos que:

$$
\sigma(v, t)=\sigma \cdot v=\sum_{i=1}^{n} v_{i} \sigma_{i}
$$

De donde

$$
\int_{S} \sigma(v) d S=\int_{S} \sigma \cdot v d S
$$

Por el Teorema de Divergencia obtenemos que,

$$
\int_{S} \sigma \cdot v d S=\int d i v \sigma d V
$$


Sustituyendo en (6), obtenemos:

$$
\int_{V} \rho \frac{d^{2}}{d t^{2}} u d V=\int_{V} d i v \sigma d V+\int_{V} \rho F d V
$$

De donde,

$$
\int\left(\operatorname{div} \sigma+\rho F-\rho \frac{d^{2}}{d t^{2}} u\right) d V=0
$$

Y como V es arbitrario, una condición necesaria y suficiente para el balance lineal del momento es la ecuación:

$$
\operatorname{div} \sigma+\rho F=\rho \frac{d^{2}}{d t^{2}} u
$$

La ecuación (7) es llamada Primera Ley del Movimiento de Cauchy.

\section{FORMULACIÓN DEL PROBLEMA DINÁMICO}

Consideremos un cuerpo homogéneo localizado en una región $\Omega \subseteq R^{n}$. Sea $\mathrm{T}>0$, $Q=\Omega \times] 0, T\left[, \sum=\Gamma \times\right] 0, T[$ la frontera lateral del cilindro $Q$.

Sea $u=u(x, t)$ función escalar o desplazamiento con respecto a un estado relajado. Denotemos con,

$$
\sigma=\left(\sigma_{1}, \sigma_{2}, \ldots, \sigma_{n}\right) \quad \varepsilon=\left(\varepsilon_{1}, \varepsilon_{2}, \ldots, \varepsilon_{n}\right)
$$

Supondremos que el cuerpo está fijo en los extremos, es decir, satisface las condiciones de frontera,

$$
u=0 \quad \text { sobre } \sum
$$

y que satisface las condiciones iniciales,

$$
u(x, 0)=u_{0}(x) ; \quad u_{t}(x, 0)=u_{1}(x), \quad \text { en } \Omega
$$

y las condiciones de historia nula

$$
u(x, t)=0, \quad t \leq 0 .
$$

Para nuestro caso, de (1) obtenemos que la deformación es dada por

$$
\varepsilon_{i}(x, t)=\frac{\partial u}{\partial x_{i}} \quad(i=1,2, \ldots, n) .
$$


Si la propiedad del material es lineal y la tensión actúa en una parte de la frontera $\Gamma$, entonces de (4) la relación constitutiva tensión - deformación es dada por,

$$
\sigma_{i}(x, t)=G(0) \varepsilon_{i}(x, t)-\int_{0}^{t} g(\tau) a(x) \varepsilon_{i}(x, t-\tau) d \tau, \quad i=1, \ldots, n .
$$

Para desplazamientos uniformes, la ecuación del balance del momento lineal de (7) es dada por:

$$
\operatorname{div} \sigma+\rho F=\rho \frac{d^{2}}{d t^{2}} u
$$

Derivando (9) con respecto a $x_{i}$ y sumando en $i$, tenemos:

$$
\sum_{i}^{\partial \sigma_{i}}=G(0) \sum_{i} \partial x_{i} \varepsilon_{i}-\int_{b}^{t} g(\tau) \sum_{i} \frac{\partial}{\partial x_{i}}\left(a(x) \varepsilon_{i}(x, t-\tau)\right) d \tau
$$

De (8) y (10) y la propiedad de la divergencia tenemos:

$$
\begin{aligned}
& \sum_{i}^{\partial \sigma_{i}}=\operatorname{div} \sigma=\rho u_{i}-\rho F, \quad \sum_{i}^{\partial \varepsilon_{i}}=\sum_{i} \frac{\partial^{2}}{\partial x_{i}^{2}} u=\Delta u, \quad \mathrm{y} \\
& \sum_{i} \frac{\partial}{\partial x_{i}}\left(a(x) \varepsilon_{i}\right)=a \Delta u+\nabla a \cdot \nabla u=\operatorname{div}\{a \nabla u\} .
\end{aligned}
$$

De donde podemos obtener (11) como,

$$
\rho u_{t t}=\rho F+G(0) \Delta u-\int_{0}^{t} g(\tau) d i v\{a(x) \nabla u(t-\tau)\} d \tau .
$$
sistema:

Por simplicidad, se considera $\rho=1, \quad F \equiv 0 \quad$ y $\quad G(0)=1 ; \quad$ obteniéndose el

$$
\begin{aligned}
& u_{t t}-\Delta u+\int_{0}^{t} g(t-\tau) \operatorname{div}\{a(x) \nabla u(\tau)\} d \tau=0 \text { en } Q \\
& u=0 \text { sobre } \sum \\
& \begin{array}{ll}
u(x, 0)=u_{0}(x), u_{t}(x, 0)=u_{1}(x) & \text { en } \Omega \\
u(x, t)=0, t \leq 0 . &
\end{array}
\end{aligned}
$$

Observe que de (5), obtenemos

$$
1-\int_{0}^{t} a(x) g(t-\tau) d \tau>0, \quad x \in \Omega, t>0 .
$$

A este sistema se le aplica diversas Técnicas del análisis funcional para obtener su correspondiente solución global y otras informaciones adicionales. 


\section{BIBLIOGRAFÍA}

1. BOLTZMANN, L., ZUR theorie der elastishen Nachwirkung. Ann. Phys.,7 (1986), Erganzungsband pp 624-625.

2. FERRY,J.D., Viscoelastic Properties of Polimers. John Wiley and Sons Inc, $3^{\text {th }}$ Ed. (1980)

3. FABRIZIO, M.- MORRO, A., Mathematical problemas in linear viscoelasticity. SIAM. Studies in Applied Mathematics, Vol 12, Philadelphia (1992).

4. FINDLEY, W.N.- LAI,J.S.-ONARAN, K., Creep and Relaxation of Nonlinear, viscoelastic materials. Series in Appl. Math. And Mech. Vol 18, North-Holland, Amsterdam, (1976).

5. GRASELLI, M., Determining the relaxation tensor in linear viscoelasticity of integral, type. Publ. IAN CNR; Pavia, 829 pp 1-22 (1992).

6. GREEN, A. E. RIVLIN, R.S., The mecanism of nonlinear materials with memory. Part I, Arch. Rat. Mech. Anal. 1, pp 1-21 (1,957); Part III, Arch. Rat. Mech. Anal. 1, pp387- 404 (1960).

7. GREENBER G, J.M.- LI TATSIEN, The effect of the boundary damping for the quasilinear wave equation. J. Differential Equations 52 (1), pp 66-75 (1984).

8. HRUSA, W.J.- NOHEL, J.A., The Cauchy problem in one dimensional nonlinear viscoelasticity. J. Differential Equations 58 (1), pp 388-412 (1985). 